\title{
$\checkmark$ Research Square \\ Identification of Molecular and Immune Prognostic Classifiers of Acute Myeloid Leukemia
}

Fang Zhou

Southeast University

Baoan Chen ( $\nabla$ cba8888@hotmail.com )

Southeast University

\section{Research Article}

Keywords: acute myeloid leukemia, bioinformatic analysis, AML microenvionment, prognosis, relapse

Posted Date: February 15th, 2021

DOl: https://doi.org/10.21203/rs.3.rs-171395/v1

License: (9) This work is licensed under a Creative Commons Attribution 4.0 International License. Read Full License 


\section{Abstract \\ Background}

Acute myeloid leukemia (AML) is a clinically and genetically heterogeneous hematological malignancy and relapse is the main reason for the poor therapeutic effect and low survival rate. Bioinformatic technology could screen out relative genes that promote the recurrence of $A M L$, providing a theoretical basis for further improving the precision stratification treatment of AML.

\section{Methods}

In this study, gene expression profiles of Dataset Acute Myeloid Leukemia (OHSU, Nature 2018) and GSE134589 were downloaded from cbioportal and GEO, respectively. R software and limma packages were used to identify the DEGs and then run GO enrichment, KEGG pathway, and PPI network. CIBERSORTx was used to enumerate tumor-infiltrating immune cells. Prognosis-related genes were selected by Univariate and multivariate Cox proportional hazards regression analyses and the expression of them were verified by GEPIA. Kaplan-Meier curve analysis could compare the survival time. ROC curve analysis was performed to predict the value of the selected genes.

\section{Results}

Functional analysis showed that the up-regulated DEGs were strikingly enriched in Cytokine-cytokine receptor interaction and positive regulation of cytokine production, and the down-regulated DEGs in the regulation of cell-cell adhesion, TNF signaling pathway. CIBERSORTx analysis revealed that the immune response of $A M L$ acted as an intricate network and proceeded in a tightly regulated way. Cox analysis showed that ALDH1L2, KLK1, and LRRN2 were correlated with AML prognosis.

\section{Conclusions}

ALDH1L2, KLK1, and LRRN2 are prognosis-related genes in AML, which may together with some immune pathways, induce poor prognosis, and can be used as potential biomarkers in AML treatment.

\section{Introduction}

Acute myeloid leukemia (AML) is a clinically and genetically heterogeneous disease characterized by clonal malignant proliferation of myeloid progenitors in the hematopoietic system, resulting in the accumulation of leukemic blasts in the bone marrow (BM) and blood(1). Traditional chemotherapy combined with hematopoietic cell transplantation (HCT) as a fundamental strategy for AML has changed significantly in the past few years $(2,3)$,on account of the U.S. Food \& Drug Administration (FDA) has approved several new drugs for AML】including GlasdegibロVenetoclax, and others(4-7). Notwithstanding 
the above, deaths due to disease persistence and recurrence remain constant, and overall survival for AML patients remains poor. About $20 \%-30 \%$ of patients never achieve complete remission (CR) after firstline intensive treatment and $50 \%$ relapse after CR accomplishment $(1,8)$.

In recent years, in addition to the advent of new drugs that profoundly affect the treatment landscape of $\mathrm{AML}$, the use of cytogenetic abnormalities and gene mutations for risk stratification and prognosis assessment has also been further improved with the deepening understanding of the pathogenesis of AML(9-12). The 2017 edition of the European LeukemiaNet (ELN) recommendations for diagnosis and management of $A M L$ in adults, including a revised version of the risk stratification by genetics. For example, monosomal karyotype, RUNX1, ASXL1, and TP53 mutations have been added as features of the adverse-risk group due to their independent associations with risk (13-17). However, according to this proposal, approximately $30 \%$ of AML patients are still listed as having intermediate-risk, many of whom do not carry prognostic nuclear abnormalities or gene mutations, and the choice of the ideal strategy remains unclear. Therefore, the current risk stratification and prognosis assessment of AML patients need to be further improved. Considering that the incidence of AML relapse remains one of the major problems in $A M L$ treatment and that patients with relapsed $A M L$ respond less to treatment and have a poorer overall survival. we selected 1298 adult AML patients from TCGA and GEO datasets, including those with denovo $A M L$ and relapsed $A M L$ patients,to screen relapse-related genes and explore their influence on the prognosis of AML patients, so as to provide a theoretical basis for hierarchical management of relapsed AML.

Notably, AML is composed not only of leukemic cells but also of non-leukemic cells, in which the immune microenvironment may play an important role in promoting the development and recurrence of $\mathrm{AML}(18)$. In recent years, several promising preclinical and clinical immunotherapies, including immune checkpoint inhibitors and active or passive immunotherapies, have shown promising results in a variety of cancers, but the effect is not obvious for $A M L$, suggesting that $A M L$ has a unique mechanism to evade treatment (19-23). To better understand the role of immunity in AML, we summarized the number of immune cells in AML from the perspective of the diversity and nature of infiltrating immune cells in AML. CIBERSORTX is a multifunctional transfer algorithm based on gene expression that quantifies cell components from gene expression profiles of large tissues(24). In this study, we used CIBERSORTx to enumerate 22 different functional immune cell types in AML to define the landscape of relapse AML and denovo AML. More importantly, we studied its relationship and survival with other immune cells. We hope this study will provide some important information about the complex mechanisms in the incidence of AML relapse and help to improve treatment strategies for AML.

\section{Material And Methods}

\section{Dataset Collection}

Gene expression profile of Dataset GSE134589 was downloaded from Gene Expression Omnibus (GEO) database (https://www.ncbi.nlm.nih.gov/geo/query/acc.cgi?acc=GSE134589). This dataset contains the 
RNA-sequencing profiling of 432 acute myeloid leukemia samples. Except for complete remission patients, we included 368 diagnosis samples and 35 relapsed samples to compare the mRNA levels of relapsed patients and those with Devono. Gene expression profile of Dataset Acute Myeloid Leukemia (OHSU, Nature 2018) was downloaded from cBioportal for Cancer Genomics (cbioportal) database(http://www.cbioportal.org/study/summary?id=aml_ohsu_2018). This dataset contains 672 acute myeloid leukemia samples (with 454 matched normals) and both provided RNA sequencing data and clinical information. A total of 266 Devono and 40 relapsed AML patients were included in the analysis, excluding M3 leukemia (according to the French-American-British (FAB) classification systems).

\section{Identification of Differentially Expressed mRNAs (DEGs) in Relapsed and Devono AML patients}

Ensemble BioMart databank GRCh37.p13 version supplied annotations to selected genes,and differentially expressed genes (DEGs) between relapsed and Devono patients were defined as those genes with $\| \log 2 \mathrm{FCl}>1.5$, $\mathrm{p}$-value $<0.05$. Package "limma" was used to screen out the DEGs between relapsed and Devono AML samples. Volcano plot was completed by "ggpubr" and "ggthemes" package in R 4.0.1.

\section{Functional and Pathway Enrichment Analysis}

To examine a complete collection of functional annotations of DEGs, Gene Ontology (GO) term enrichment analysis and Kyoto Encyclopedia of Genes and Genomes (KEGG) pathway analysis were performed by using the "cluster Profiler" R package. The "cluster Profiler" R package was also used for the visualization of GO and KEGG results. p-value $<0.05$ was set to be significant. "Cluster Profiler" is a package of "Bioconductor", it implements methods to investigate and visualize functional profiles (GO and KEGG) of gene and gene clusters. The download address is (http://bioconductor.org/packages/release/bioc/html/clusterProfiler.html).

\section{Protein-Protein Interaction Network (PPI) Analysis and Visualization}

The STRING online database (https://string-db.org/cgi/input.pl) provided PPI (protein-protein communication) information. We used STRING database to investigate the interactions between DEGs and visualize the results using Cytoscape software. Cytoscape MCODE plug-in provided access to select the significant modular of PPI network. The patterns default parameters as follows: node density cutoff = 0.1 , node score cutoff $=0.2$, degree cut off $=2, k$-core $=2$, and max. depth $=100$. For genes in the hub module, we use ClueGO + CluePedia for functional enrichment analysis.

\section{Enumeration of tumor-infiltrating immune cells}

CIBERSORTx is a powerful immune infiltrate analysis tool that can distinguish between each immune cell subtype and precisely calculate and quantify different immune cell proportions. The obtained p-value indicates the statistical significance and can help filter out samples with insignificant efficiency. Before 
running CIBERSORTx, the gene expression data downloaded from cbioportal and GEO datasets must be processed as described by Binbin Chen et al(25). Then, the data was uploaded to the CIBERSORTX internet portal(https://CIBERSORTx.stanford.edu/runCIBERSORTx.php) with some permutations being set to 100. Related proportions of 22 infiltrating immune cells together with CIBERSORTx metrics of CIBERSORTX P-value, Pearson correlation coefficient, and root mean squared error (RMSE) were evaluated for each sample concurrently. Samples with $\mathrm{P}<0.05$ in CIBERSORTx analysis result were used in further analysis. Mann-Whitney $U$ test was used to compare differences in immune cell subtypes in relapsed and denovo groups.

\section{Construction of a prognostic signature}

Univariate Cox proportional hazards regression analysis was performed to investigate the influence of each gene on overall survival. With the cutoff of $p<0.05$, DEGs were considered to be prognosis-related. Multivariate Cox proportional hazards regression analysis was further performed on the prognosis-related genes. Cox proportional hazards regression with a $p<0.05$ was set for risk score of developing relapsed in AML patient. According to the mean risk score, patients were divided into low-and- high-risk groups. The Kaplan-Meier curve analysis was further conducted to estimate the relationship between the risk score and overall survival. $p<0.05$ was a significant cutoff. Time-dependent receiver operating characteristic $(\mathrm{ROC})$ curve analysis was also performed to evaluate the 4-year predictive value of the outcomes, and the area under the curve (AUC) of the ROC curve was calculated to examine the classifier performance.

\section{Validation of real prognosis-related genes}

The prognosis-related genes were finally validated in Gene Expression Profiling Interactive Analysis (GEPIA: http://gepia.cancer-pku.cn/). Differential expression of AML patients and their normal samples were compared by using GEPIA database. Genes with statistically significant differences in expression were defined as the "real" prognosis-related genes.

\section{Statistical Analysis}

The log1.5FC values of the computationally selected mRNAs were already normalized by $\mathrm{R}$ package. Student's t-test was used to compare gene expression between relapse and denovo AML groups. Univariate and multivariate Cox regressions were conducted by using the "survival" package in $\mathrm{R}$ package. The OS between different groups was compared by Kaplan-Meier analysis with the log-rank test. All statistical analyses were performed with $\mathrm{R}$ software, and statistical significance was set at probability values of $p<0.05$.

\section{Results}

\section{Identification of DEGs}


Complete clinical data and RNA sequence data of 306 non-M3 AML patients (266 patients with denovo $A M L$ and 40 patients with relapsed/refractory $A M L)$ and 403 archival BM samples $(n=368$ with denovo $A M L$ and $n=35$ with relapsed/refractory $A M L$ ) from the GEO database were contained in our present study. The subtypes were classified according to the FAB classification systems in which M3 subtype was removed from the present analysis regarding the separate properties. Through the limma software package, 451 and 55 DEGs from OHSU and GSE134589 were extracted respectively. The differential expression of varied genes from two sets of sample data included in each of the two datasets is shown in Fig. 1A囚1B. Results showed that a total of $451 \mathrm{DEGs}$ were detected in OHSU dataset, including 86 downregulated genes $(\log F C<-1.5)$ and 365 up-regulated genes $(\log F C>1.5)$, and 55 DEGs were identified in GSE134589, including 18 downregulated genes ( $\log \mathrm{FC}<-1.5)$ and 37 up-regulated genes $(\log F C>1.5)$.

\section{Functional and Pathway Enrichment Analysis of DEGs}

The results of functional and pathway enrichment analysis are shown in Fig. 2A-2E. Gene Ontology (GO) analysis of up-regulated DEGs in OHSU showed that DEGs were related to leukocyte migration, the regulation of ion transmembrane transport, negative regulation of cell activation, $T$ cell-mediated immunity, and positive regulation of interleukin-8. Gene Ontology (GO) analysis of down-regulated DEGs of OHSU showed no functional or pathway enriched. Gene Ontology (GO) analysis of down-regulated DEGs of GSE134589 dataset showed that regulation of cell-cell adhesion, leukocyte proliferation, positive regulation of $\mathrm{T}$ cell activation, and proliferation events were mostly enriched. Among the functional and pathway enrichment analysis, the cytokine-cytokine receptor interaction events, TNF-signaling pathway, and RIG--llike receptor signaling pathway were enriched most. Gene Ontology (GO) analysis of upregulated DEGs of GSE134589 dataset showed that positive regulation of cytokine production, leukocyte migration, lymphocyte and mononuclear cell proliferation, as well as cytokine biosynthetic and metabolic process, were mostly enriched. Among the functional and pathway enrichment analysis, cytokine-cytokine receptor interaction, hematopoietic cell lineage, viral protein interaction with cytokine and cytokine receptor,Toll-like receptor signaling pathway, and Th17 cell differentiation were enriched most.

\section{PPI network and analysis on clusters}

STRING mapped 451 DEGs of OHSU and 55 DEGs of GSE134589 into two PPI networks containing $278 \square$ 54 nodes and $863 \otimes 352$ edges, respectively. (Fig. 3A囚3B). Then MCODE was used to discover clusters in the network. Four clusters (the top two significant modules of each dataset) were selected for GO term and KEGG pathway enrichment analysis. Among them, cluster 1 of OHSU contained 22 nodes and 102 edges, with the highest score (Fig. 3C), cluster 2 contained 11 nodes and 25 edges (Fig. 3D), cluster1 of GSE134589 contained 20 nodes and 66 edges (Fig. 3E), cluster 2 of GSE134589 contained 13 nodes and 58 edges (Fig. 3F). We then performed a functional analysis for the 4 important clusters using ClueGO. The results shows that the positive regulation of mononuclear cell proliferation, leukocyte migration, cytokine biosynthetic proces, lymphocyte proliferation, phospholipase activity, and T cell costimulation enents were mostly enriched(Fig. 4). 


\section{The landscape of immune infiltration in relapsed and denovo AML patients}

We first exhibited the landscape of 22 immune cell subpopulations infiltration in $A M L$, and subsequently, we investigated the difference between relapsed and denovo patients using the CIBERSORTX algorithm. The portion of immune cells varied distinctly between groups (Fig. 5A, 5B). Compared with denovo AML, relapsed AML contained a greater number of Neutrophils. However, Plasma cells,T cells CD4 naive,

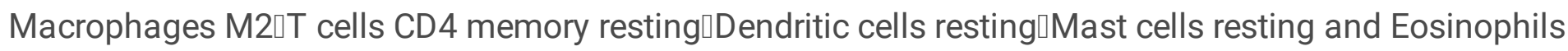
fractions were relatively lower(Table 1, Fig. 5C). The proportions of $22 \mathrm{TIIC}$ were weakly-to-strongly correlated in AML. T cells CD4 naive and T cells gamma delta showed the strongest positive correlation (Pearson correlation $=0.34$ ), while T cells CD4 naive and Monocytes showed the strongest negative correlation (Pearson correlation $=0.28$ ). Altogether, these results revealed that the immune response of AML acted as an intricate network and proceeded in a tightly regulated way (Fig. 5D). 
Table 1

Comparison of 22 TIICs proportion between Denovo and Relapse AML

\begin{tabular}{|llll|}
\hline Cell type & \multicolumn{2}{l}{ CIBERSORT fraction in \% of all infiltrating immune cells } \\
& $\begin{array}{l}\text { (mean } \pm \text { SD) } \\
\text { Denovo AML Relapse AML Pvalue }\end{array}$ & \\
\hline B cells naive & $0.042 \pm 0.060$ & $0.069 \pm 0.077$ & 0.111 \\
\hline B cells memory & $0.017 \pm 0.033$ & $0.015 \pm 0.023$ & 0.741 \\
\hline Plasma cells & $0.036 \pm 0.043$ & $0.018 \pm 0.025$ & 0.002 \\
\hline T cells CD8 & $0.021 \pm 0.033$ & $0.034 \pm 0.053$ & 0.245 \\
\hline T cells CD4 naive & $0.060 \pm 0.071$ & $0.029 \pm 0.047$ & 0.006 \\
\hline T cells CD4 memory resting & $0.019 \pm 0.044$ & $0.035 \pm 0.053$ & 0.150 \\
\hline T cells CD4 memory activated & $0.005 \pm 0.011$ & $0.009 \pm 0.021$ & 0.303 \\
\hline T cells follicular helper & $0.003 \pm 0.010$ & $0.009 \pm 0.017$ & 0.124 \\
\hline T cells regulatory (Tregs) & $0.003 \pm 0.017$ & $0.007 \pm 0.023$ & 0.443 \\
\hline T cells gamma delta & $0.026 \pm 0.033$ & $0.030 \pm 0.062$ & 0.747 \\
\hline NK cells resting & $0.076 \pm 0.100$ & $0.107 \pm 0.121$ & 0.229 \\
\hline NK cells activated & $0.029 \pm 0.054$ & $0.034 \pm 0.060$ & 0.711 \\
\hline Monocytes & $0.370 \pm 0.252$ & $0.301 \pm 0.210$ & 0.136 \\
\hline Macrophages M0 & $0.009 \pm 0.019$ & $0.011 \pm 0.037$ & 0.801 \\
\hline Macrophages M1 & $0.017 \pm 0.045$ & $0.018 \pm 0.054$ & 0.890 \\
\hline Macrophages M2 & $0.015 \pm 0.029$ & $0.005 \pm 0.013$ & 0.002 \\
\hline Dendritic cells resting & $0.002 \pm 0.001$ & $0.001 \pm 0.003$ & 0.788 \\
\hline Dendritic cells activated & $0.008 \pm 0.027$ & $0.010 \pm 0.027$ & 0.749 \\
\hline Mast cells resting & $0.167 \pm 0.187$ & $0.122 \pm 0.180$ & 0.245 \\
\hline Mast cells activated & $0.037 \pm 0.090$ & $0.047 \pm 0.109$ & 0.684 \\
\hline Eosinophils & $0.012 \pm 0.037$ & $0.001 \pm 0.002$ & $<0.001$ \\
\hline Neutrophils & $0.025 \pm 0.054$ & $0.088 \pm 0.144$ & 0.045 \\
\hline Significance of bold values are $p<0.05$. & & \\
\hline
\end{tabular}

Identification of clinical implications of TIICs subsets 
Owning to the missing survival data in included GEO datasets, we investigated whether there was a statistical relationship between specific TIICs and AML overall survival obtained from OHSU by univariate Cox regression using the survival $R$ package. After a restriction of CIBERSORTx filter to $P<0.05$, there were 53 patients with available data on overall survival. Only two meaningful TIICs subsets were selected. $T$ cells CD4 memory activated $(H R=22492961.769 ; z=2.130 ; P=0.033)$ and Macrophages M2 $(H R=$ $1575810.697 ; z=1.983 ; P=0.047$ ) were both significantly associated with a poor outcome (Fig. $6 \mathrm{~A})$. The corresponding Kaplan-Meier curve is depicted in Fig. 6B. The distribution of risk score, survival status was also analyzed (Fig. 6C).

\section{Identification of prognostic signature}

Of the OHSU data containing patient clinical information with 451 DEGs, the univariate Cox proportional hazards regression analysis screened out 27 prognostic-related genes,we then used the Gepia database to verify the expression levels of obtained genes and screened 5 genes were significantly different between AML patients and healthy controls (Fig. 7 ); multivariate Cox proportional hazards regression analysis was further performed on the 5 genes, which screened ALDH1L2, KLK1, and LRRN2. The risk score for predicting overall survival was calculated as follows: Risk score $=0.344 * A L D H 1 L 2+0.309$ * KLK1 + 0.254* LRRN2 (Fig. 8A). According to the risk score, patients were divided into low- and high-risk groups. Survival analysis showed that low-risk patients had longer overall survival than high-risk patients (Fig. 8B). The distribution of risk score, survival status was shown in Fig. 8C. The AUC of 4-year survival TIME ROC curve analysis was 0.73 (Fig. 8D).

\section{Discussion}

In this study, we screened out AML relapse-related DEGs based on TCGA and GSE134589 datasets. The up-regulated DEGs were strikingly enriched in the immune signaling pathway, Cytokine-cytokine receptor interaction, Toll-like receptor signaling pathway, and Th17 cell differentiation, and the down-regulated DEGs in Cytokine-cytokine receptor interaction, Tumor necrosis factor (TNF)-signaling pathway and RIG-Ilike receptor signaling pathway. We further screened out 332 hub DEGs, most of which were enriched in the positive regulation of mononuclear cell proliferation, leukocyte migration, cytokine biosynthetic proces, lymphocyte proliferation, phospholipase activity, and T cell costimulation enents.

Cytokine-cytokine receptor interaction plays a significant role in the pathogenesis of various types of lymphomas (26). Toll-like receptors (TLRs) and RIG-I-like receptors (RLRs) are protective immune guards, which can initiate signaling pathways completing activation of NF-kappaB, MAP kinases, and IRFs that manage the transcription of genes encoding type I interferon and other inflammatory cytokines $(27,28)$. Previous studies have delineated that TLRs are in connection with hematopoietic damage, leading to expanded HSC proliferation and an increased bias toward myeloid cell differentiation, contributing its strength to the development of hematopoietic malignancies and bone marrow failure (29-32). This may be owing to the release of proinflammatory cytokines that could form a microenvironment accessory to promoting tumorigenesis. Th17 cells were known as a subgroup of Th cells showing proinflammatory as well as tumor-promoting qualities in diverse cancer types $(33,34)$. The TNF-signaling pathway is a 
principal mediator of apoptosis, inflammation, and immunity, and it has been implicated in the pathogenesis of a broad spectrum of human diseases, including diabetes, cancer, and different type of inflammatory diseases (35). Similarly, researches have illustrated that regulation of phospholipase activity fosters the development of tumors (36). It is not difficult to find that DEGs were mainly enriched in the immune regulation, as same as inflammation and apoptosis pathways, exhibiting proinflammatory and tumor-promoting characteristics, play an important role in generating a microenvironment conducive to the extension of tumor cells. All these findings point out the possible relationship between some DEGs and relapse AML development, which may give a new direction for relapse AML research.

Through the enrichment of DEGs, we found that most of the genes related to the recurrence of AML are gathered in the immunocorrelation pathways. Earlier studies have also told that the immune response plays an essential role in the evolution and migration of various tumors, and immunotherapy and targeted therapy are also considered as two dominant agents in tumor therapy in addition to chemotherapy. Therefore, in this study, CIBERSORTx algorithm was used to presume the percentage of 22 TIICs subsets from AML transcriptomes from OHSU and GSE134589 databases, and to reveal distinguished patterns of TIICs in initial and recurrent AML, as well as the associations between different immune cell subsets with clinical outcomes. We observed notable differences in immune cell composition between relapse AML and denovo AML. Compared with denovo AML, relapsed AML contained a greater number of Neutrophils. However, Plasma cells, T cells CD4 naive, Macrophages M2ロT cells CD4 memory resting Dendritic cells resting Mast cells resting, and Eosinophils fractions were relatively lower. Moreover, our work validated the findings that certain immune cell subsets can also predict clinical outcomes besides the Immunocore. By univariate Cox regression analysis, we found that $T$ cells CD4 memory activated and Macrophages M2 are significantly associated with poor outcome. Paradoxically, in this study, the immune cell subpopulation, which forecasted poor prognosis, did not have a significantly high expression in relapsed AML. On the contrary, Macrophages M2 expressed lower than that of denovo AML, while T cells CD4 memory activated made no significant difference. Since only two data sets were included in this study, and only 53 patients in one data set were used for immunerelated prognostic analysis, more clinical data will be needed to investigate the role of immune cell subsets in relapsed AML. It's worth noting that in multiple situations the immune function may vary greatly, although there is no difference in the number of immune cells. Subsequent studies can specifically study the functions of T cells and macrophages in the recurrence of AML.

We found the expression of ALDH1L2, KLK1, and LRRN2 were significantly different in relapse and denovo $A M L$, and were negatively related to the overall survival of the AML patients. ALDH1L2 encodes for a mitochondrial FDH and is involved in controlling the metabolic period of tetrahydrofolate (THF) in mitochondria. In recent years, the significance of mitochondrial metabolism in tumor cells has attracted more and more attention. ALDH1L2 has been shown to promote melanoma metastasis in vivo, and shorter survival has been reported in colorectal and lung adenocarcinoma studies(37-39). KLK1 belongs to the serine protease family of proteolytic enzymes, and growing evidence suggests that many KLKs are implicated in carcinogenesis(40). LRRN2 \also known as GAC1, was found overexpressed in malignant gliomas $\square$ and is a candidate for the target gene in the 1q32.1 amplicon in malignant gliomas(41). In the 
present study, we verified the prognostic value of ALDH1L2, KLK1Land LRRN2 in AML, which broadens the landscape of ALDH1L2, KLK1Iand LRRN2 research, and can guide the future exploration into relapse AML mechanism.

\section{Declarations}

\section{Acknowledgments:}

This work was supported by the [National Natural Science Foundation of China \#1] under Grant [numbers 81170492 and 81370673 ]; [National High Technology Research and Development Program 863 of People's Republic of China \#2] under Grant [number 2012AA022703]; [National Key Basic Research Program 973 of People's Republic of China \#3] under Grant [number 2010CB732404]; [Key Medical Projects of Jiangsu Province \#4] under Grant [number BE2017747]; [Key Medical of Jiangsu Province \#5] under Grant [number ZDXKB2016020] and [Jiangsu Social Development Project\#6] under Grant [number BE2018711].

\section{Conflicts of Interest:}

The authors declare no potential conflicts of interest.

\section{References}

1. Dohner H, Weisdorf DJ, Bloomfield CD. Acute Myeloid Leukemia. N Engl J Med. 2015;373(12):113652.

2. O'Dwyer K, Freyer DR, Horan JT. Treatment strategies for adolescent and young adult patients with acute myeloid leukemia. Blood. 2018;132(4):362-8.

3. Yates JW, Wallace HJ, Jr., Ellison RR, Holland JF. Cytosine arabinoside (NSC-63878) and daunorubicin (NSC-83142) therapy in acute nonlymphocytic leukemia. Cancer Chemother Rep. 1973;57(4):485-8.

4. DiNardo CD, Jonas BA, Pullarkat V, Thirman MJ, Garcia JS, Wei AH, et al. Azacitidine and Venetoclax in Previously Untreated Acute Myeloid Leukemia. N Engl J Med. 2020;383(7):617-29.

5. Kim ES. Enasidenib: First Global Approval. Drugs. 2017;77(15):1705-11.

6. Krauss AC, Gao X, Li L, Manning ML, Patel P, Fu W, et al. FDA Approval Summary: (Daunorubicin and Cytarabine) Liposome for Injection for the Treatment of Adults with High-Risk Acute Myeloid Leukemia. Clin Cancer Res. 2019;25(9):2685-90.

7. Norsworthy KJ, By K, Subramaniam S, Zhuang L, Del Valle PL, Przepiorka D, et al. FDA Approval Summary: Glasdegib for Newly Diagnosed Acute Myeloid Leukemia. Clin Cancer Res. 2019;25(20):6021-5.

8. Vasu S, Kohlschmidt J, Mrozek K, Eisfeld AK, Nicolet D, Sterling LJ, et al. Ten-year outcome of patients with acute myeloid leukemia not treated with allogeneic transplantation in first complete 
remission. Blood Adv. 2018;2(13):1645-50.

9. Byrd JC, Mrozek K, Dodge RK, Carroll AJ, Edwards CG, Arthur DC, et al. Pretreatment cytogenetic abnormalities are predictive of induction success, cumulative incidence of relapse, and overall survival in adult patients with de novo acute myeloid leukemia: results from Cancer and Leukemia Group B (CALGB 8461). Blood. 2002;100(13):4325-36.

10. Eisfeld AK, Kohlschmidt J, Mims A, Nicolet D, Walker CJ, Blachly JS, et al. Additional gene mutations may refine the 2017 European LeukemiaNet classification in adult patients with de novo acute myeloid leukemia aged $<60$ years. Leukemia. 2020;34(12):3215-27.

11. Grimwade D, Hills RK, Moorman AV, Walker H, Chatters S, Goldstone AH, et al. Refinement of cytogenetic classification in acute myeloid leukemia: determination of prognostic significance of rare recurring chromosomal abnormalities among 5876 younger adult patients treated in the United Kingdom Medical Research Council trials. Blood. 2010;116(3):354-65.

12. Marcucci G, Haferlach T, Dohner H. Molecular genetics of adult acute myeloid leukemia: prognostic and therapeutic implications. J Clin Oncol. 2011;29(5):475-86.

13. Dohner H, Estey E, Grimwade D, Amadori S, Appelbaum FR, Buchner T, et al. Diagnosis and management of AML in adults: 2017 ELN recommendations from an international expert panel. Blood. 2017;129(4):424-47.

14. Gaidzik VI, Bullinger L, Schlenk RF, Zimmermann AS, Rock J, Paschka P, et al. RUNX1 mutations in acute myeloid leukemia: results from a comprehensive genetic and clinical analysis from the AML study group. J Clin Oncol. 2011;29(10):1364-72.

15. Wong TN, Ramsingh G, Young AL, Miller CA, Touma W, Welch JS, et al. Role of TP53 mutations in the origin and evolution of therapy-related acute myeloid leukaemia. Nature. 2015;518(7540):552-5.

16. Kakosaiou K, Panitsas F, Daraki A, Pagoni M, Apostolou P, loannidou A, et al. ASXL1 mutations in AML are associated with specific clinical and cytogenetic characteristics. Leuk Lymphoma. 2018;59(10):2439-46.

17. Schnittger S, Eder C, Jeromin S, Alpermann T, Fasan A, Grossmann V, et al. ASXL1 exon 12 mutations are frequent in $A M L$ with intermediate risk karyotype and are independently associated with an adverse outcome. Leukemia. 2013;27(1):82-91.

18. Hanahan D, Weinberg RA. Hallmarks of cancer: the next generation. Cell. 2011;144(5):646-74.

19. Hartnett EG, Knight J, Radolec M, Buckanovich RJ, Edwards RP, Vlad AM. Immunotherapy Advances for Epithelial Ovarian Cancer. Cancers (Basel). 2020;12(12).

20. Kubo T, Watanabe H, Ninomiya K, Kudo K, Minami D, Murakami E, et al. Immune checkpoint inhibitor efficacy and safety in older non-small cell lung cancer patients. Jpn J Clin Oncol. 2020;50(12):144753.

21. Masarova L, Kantarjian H, Ravandi F, Sharma P, Garcia-Manero G, Daver N. Update on Immunotherapy in AML and MDS: Monoclonal Antibodies and Checkpoint Inhibitors Paving the Road for Clinical Practice. Adv Exp Med Biol. 2018;995:97-116. 
22. Ruzicka M, Koenig LM, Formisano S, Boehmer DFR, Vick B, Heuer EM, et al. RIG-I-based immunotherapy enhances survival in preclinical AML models and sensitizes AML cells to checkpoint blockade. Leukemia. 2020;34(4):1017-26.

23. Song X, Qi W, Guo J, Sun L, Ding A, Zhao G, et al. Immune checkpoint inhibitor combination therapy for gastric cancer: Research progress. Oncol Lett. 2020;20(4):46.

24. Newman AM, Steen CB, Liu CL, Gentles AJ, Chaudhuri AA, Scherer F, et al. Determining cell type abundance and expression from bulk tissues with digital cytometry. Nat Biotechnol. 2019;37(7):77382.

25. Chen B, Khodadoust MS, Liu CL, Newman AM, Alizadeh AA. Profiling Tumor Infiltrating Immune Cells with CIBERSORT. Methods Mol Biol. 2018;1711:243-59.

26. Hsu SM, Waldron J, Xie SS, Hsu PL. Hodgkin's Disease and Anaplastic Large Cell Lymphoma Revisited. 1. unique cytokine and cytokine receptor profile distinguished from that of non-hodgkin's lymphomas. J Biomed Sci. 1995;2(4):302-13.

27. Kawai T, Akira S. Toll-like receptor and RIG--like receptor signaling. Ann N Y Acad Sci. 2008;1143:120.

28. Newton K, Dixit VM. Signaling in innate immunity and inflammation. Cold Spring Harb Perspect Biol. 2012;4(3).

29. Boettcher S, Manz MG. Regulation of Inflammation- and Infection-Driven Hematopoiesis. Trends Immunol. 2017;38(5):345-57.

30. Esplin BL, Shimazu T, Welner RS, Garrett KP, Nie L, Zhang Q, et al. Chronic exposure to a TLR ligand injures hematopoietic stem cells. J Immunol. 2011;186(9):5367-75.

31. Monlish DA, Bhatt ST, Schuettpelz LG. The Role of Toll-Like Receptors in Hematopoietic Malignancies. Front Immunol. 2016;7:390.

32. Schuettpelz LG, Borgerding JN, Christopher MJ, Gopalan PK, Romine MP, Herman AC, et al. G-CSF regulates hematopoietic stem cell activity, in part, through activation of Toll-like receptor signaling. Leukemia. 2014;28(9):1851-60.

33. Chung AS, Wu X, Zhuang G, Ngu H, Kasman I, Zhang J, et al. An interleukin-17-mediated paracrine network promotes tumor resistance to anti-angiogenic therapy. Nat Med. 2013;19(9):1114-23.

34. Numasaki M, Fukushi J, Ono M, Narula SK, Zavodny PJ, Kudo T, et al. Interleukin-17 promotes angiogenesis and tumor growth. Blood. 2003;101(7):2620-7.

35. Chen G, Goeddel DV. TNF-R1 signaling: a beautiful pathway. Science. 2002;296(5573):1634-5.

36. Martins M, McCarthy A, Baxendale R, Guichard S, Magno L, Kessaris N, et al. Tumor suppressor role of phospholipase C epsilon in Ras-triggered cancers. Proc Natl Acad Sci U S A. 2014;111(11):423944.

37. Krupenko NI, Dubard ME, Strickland KC, Moxley KM, Oleinik NV, Krupenko SA. ALDH1L2 is the mitochondrial homolog of 10-formyltetrahydrofolate dehydrogenase. J Biol Chem. 2010;285(30):23056-63. 
38. Koseki J, Konno M, Asai A, Colvin H, Kawamoto K, Nishida N, et al. Enzymes of the one-carbon folate metabolism as anticancer targets predicted by survival rate analysis. Sci Rep. 2018;8(1):303.

39. Piskounova E, Agathocleous M, Murphy MM, Hu Z, Huddlestun SE, Zhao Z, et al. Oxidative stress inhibits distant metastasis by human melanoma cells. Nature. 2015;527(7577):186-91.

40. Adamopoulos PG, Kontos CK, Scorilas A. Discovery of novel transcripts of the human tissue kallikrein (KLK1) and kallikrein-related peptidase 2 (KLK2) in human cancer cells, exploiting NextGeneration Sequencing technology. Genomics. 2019;111(4):642-52.

41. Almeida A, Zhu XX, Vogt N, Tyagi R, Muleris M, Dutrillaux AM, et al. GAC1, a new member of the leucine-rich repeat superfamily on chromosome band 1q32.1, is amplified and overexpressed in malignant gliomas. Oncogene. 1998;16(23):2997-3002.

\section{Figures}

Figure $1 \mathrm{~A}$
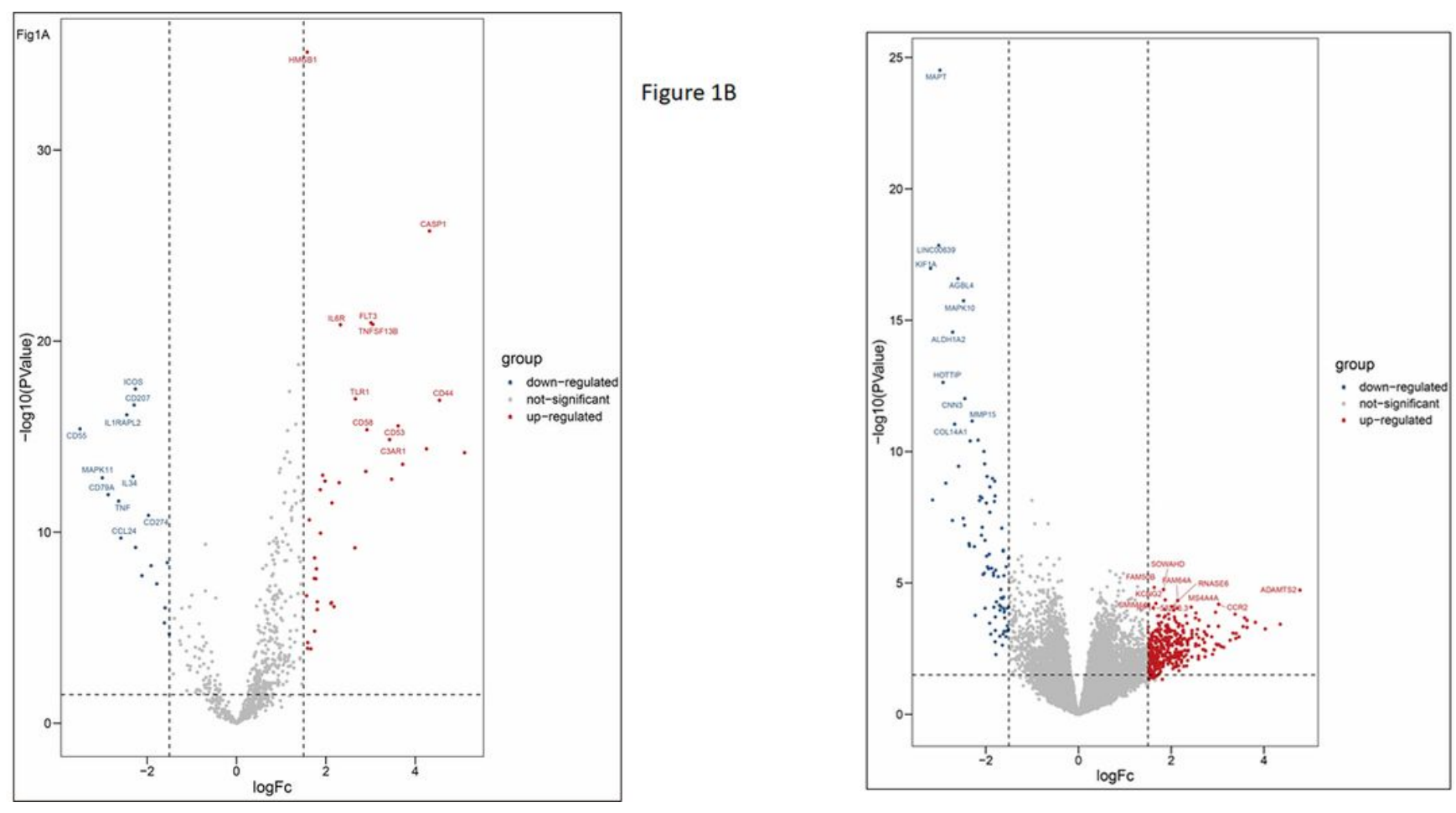

\section{Figure 1}

Volcanic maps of all genes. A. Volcanic maps of GSE134589 dataset; B. Volcanic maps of OHSU dataset. Red spot, the expression is up-regulated; Blue spot, the expression is down-regulated; Gray spot, no significantly dysregulated. 


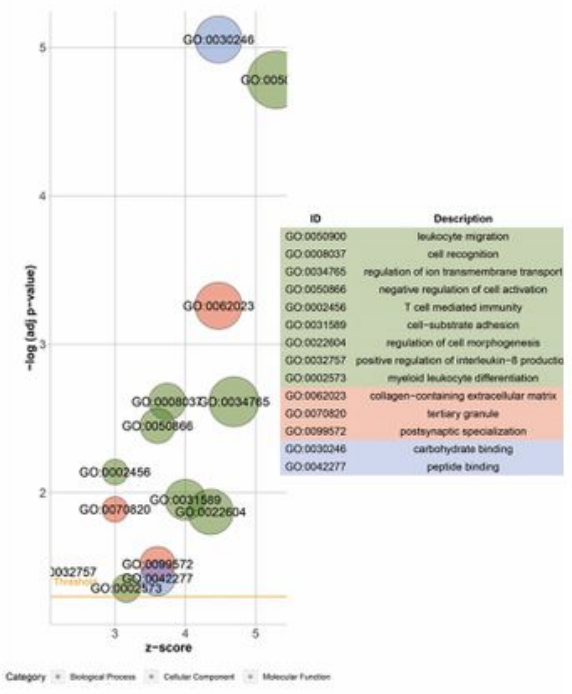

Figure 2B

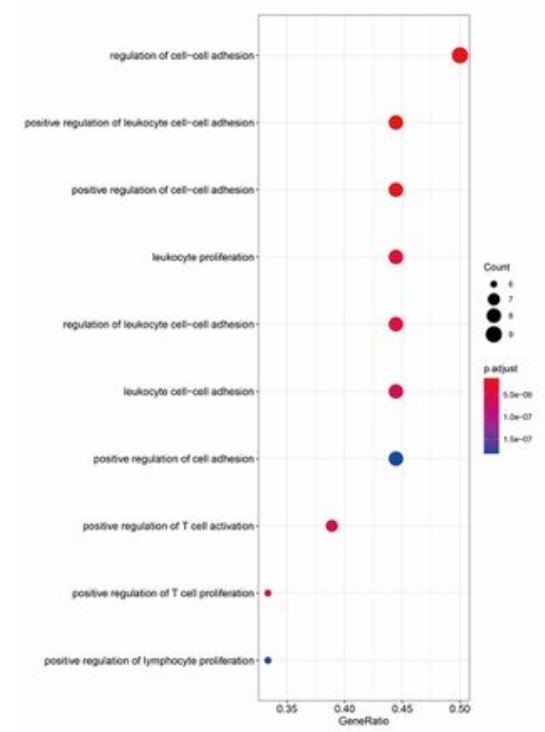

Figure $2 \mathrm{C}$
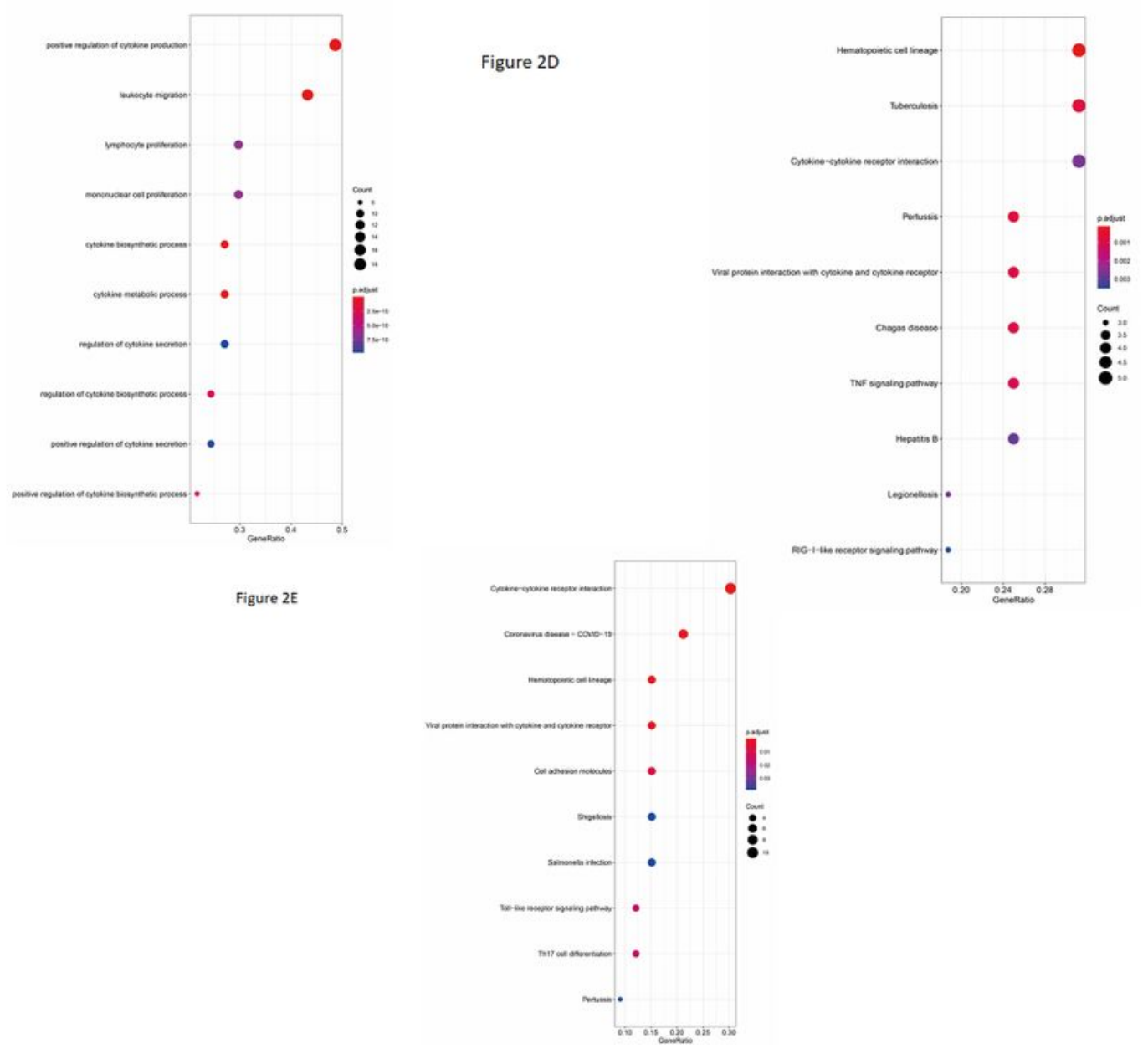

Figure 2

Functional and Pathway Enrichment Analysis of DEGs. A. The GO biological process enrichment analysis of up-regulated DEGs of OHSU. B-E The bubble chart showed the top 10 pathways with significant difference of GSE134589. B. The GO biological process enrichment analysis of down-regulated DEGs. C. The GO biological process enrichment analysis of up-regulated DEGs. D. The KEGG enrichment analysis of down-regulated DEGs. E. The KEGG enrichment analysis of up-regulated DEGs. 


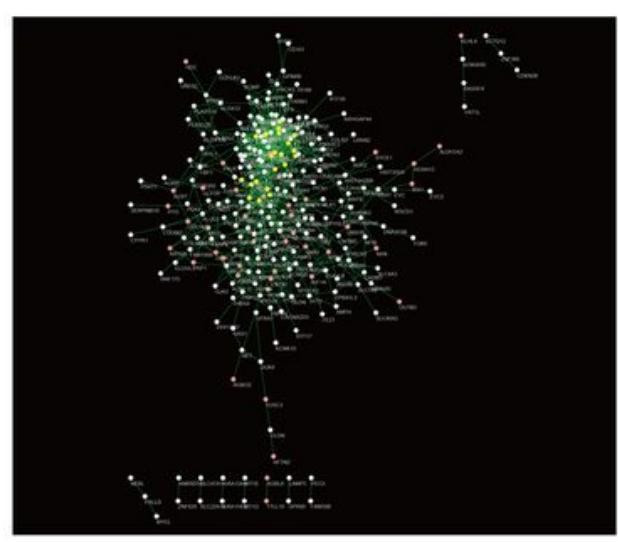

Figure 3A

Figure $3 C$

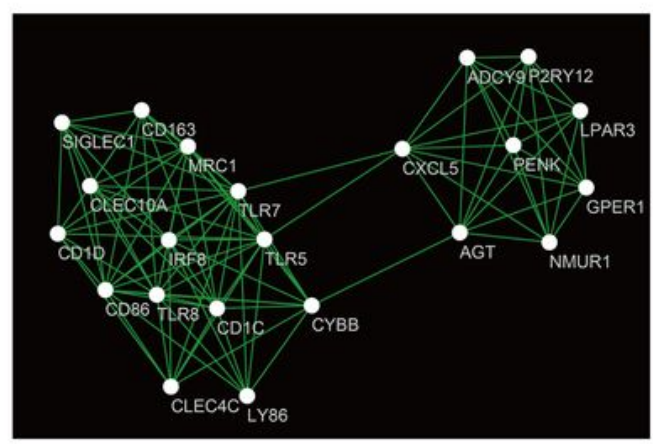

Figure 3D

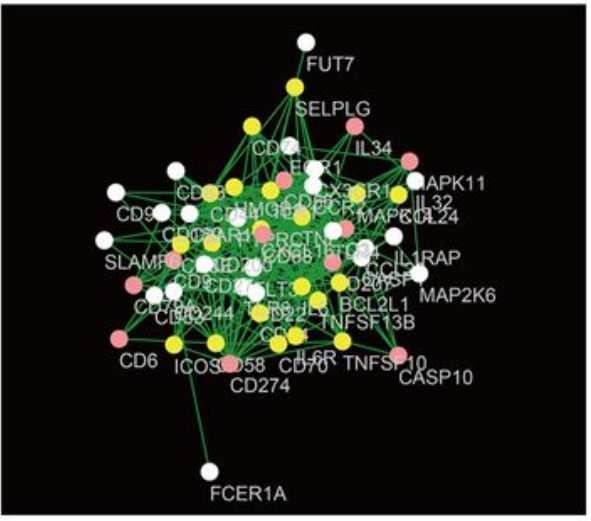

Figure 3B

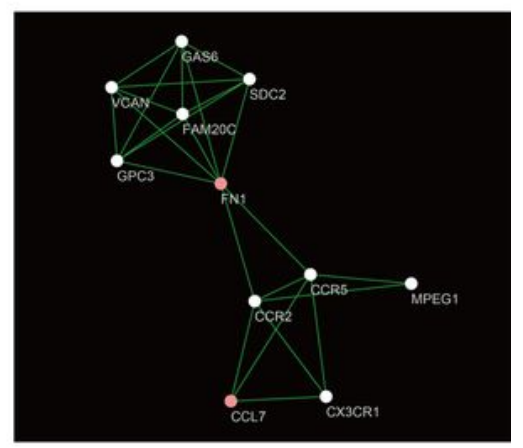

Figure 3E

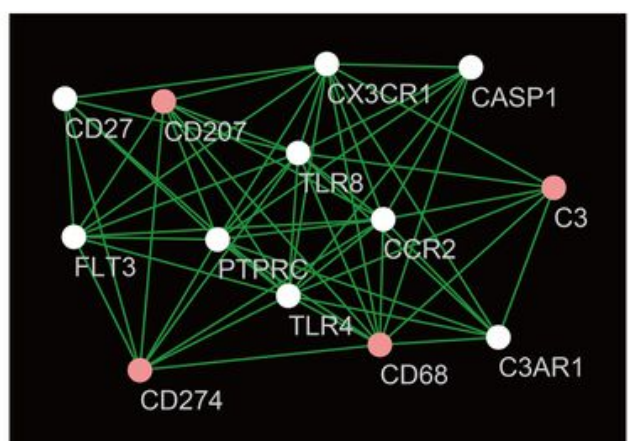

Figure 3F

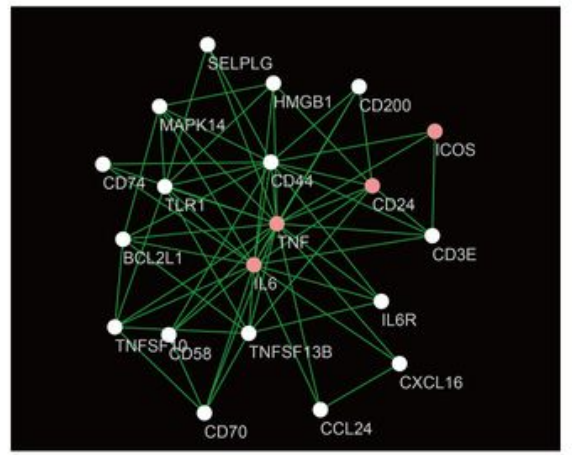

Figure 3

A. The PPI analysis of OHSU at STRING (https://string-db.org). B. The PPI analysis of GSE134589 at STRING. C₫D. Cluster analysis of the OHSU PPI network (C. Cluster1; D. Cluster2). EøF. Cluster analysis of the GSE134589 PPI network (E. Cluster1; F. Cluster2). 


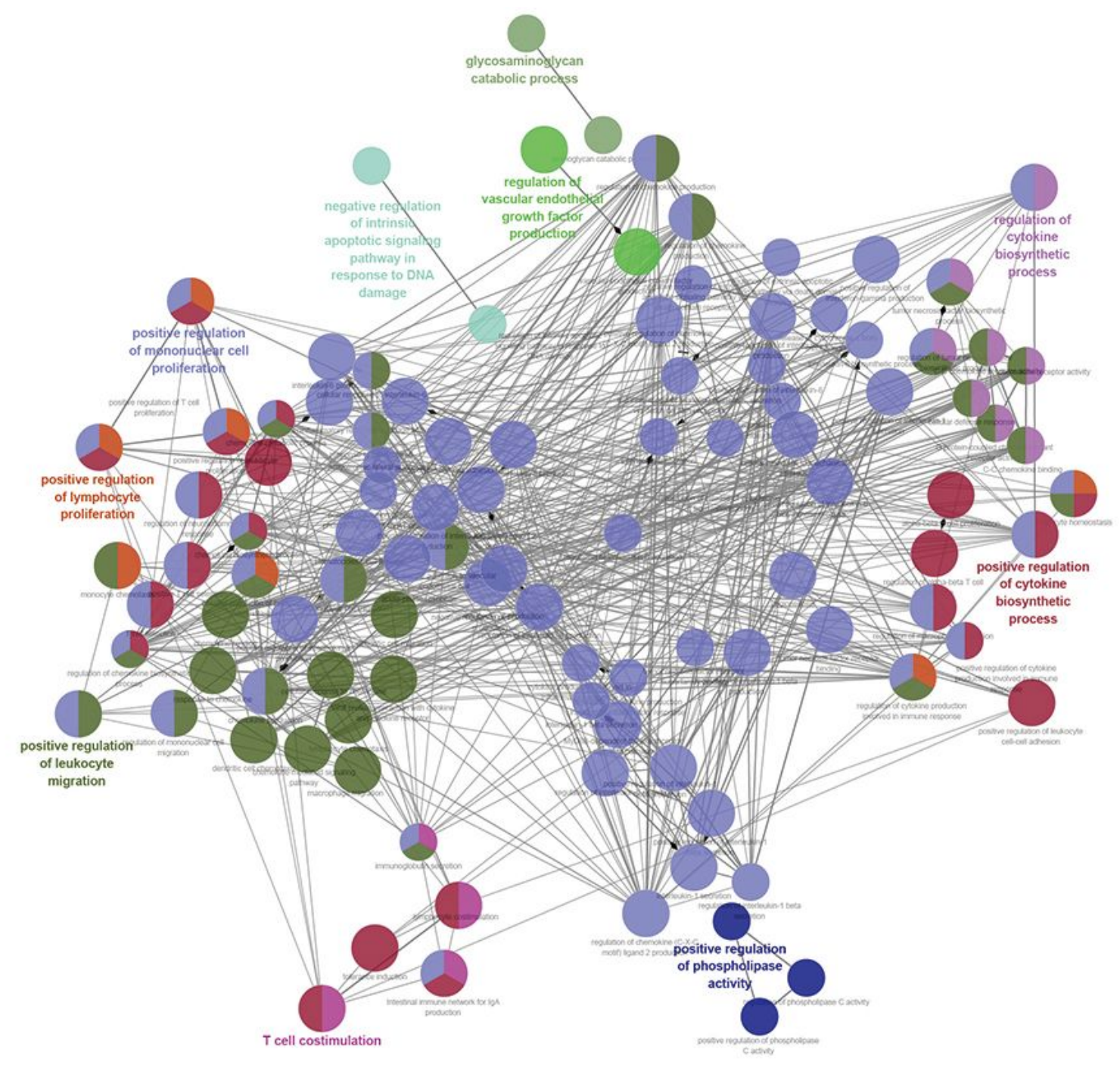

Figure 4

GO and KEGG analyses on the hub modules. 


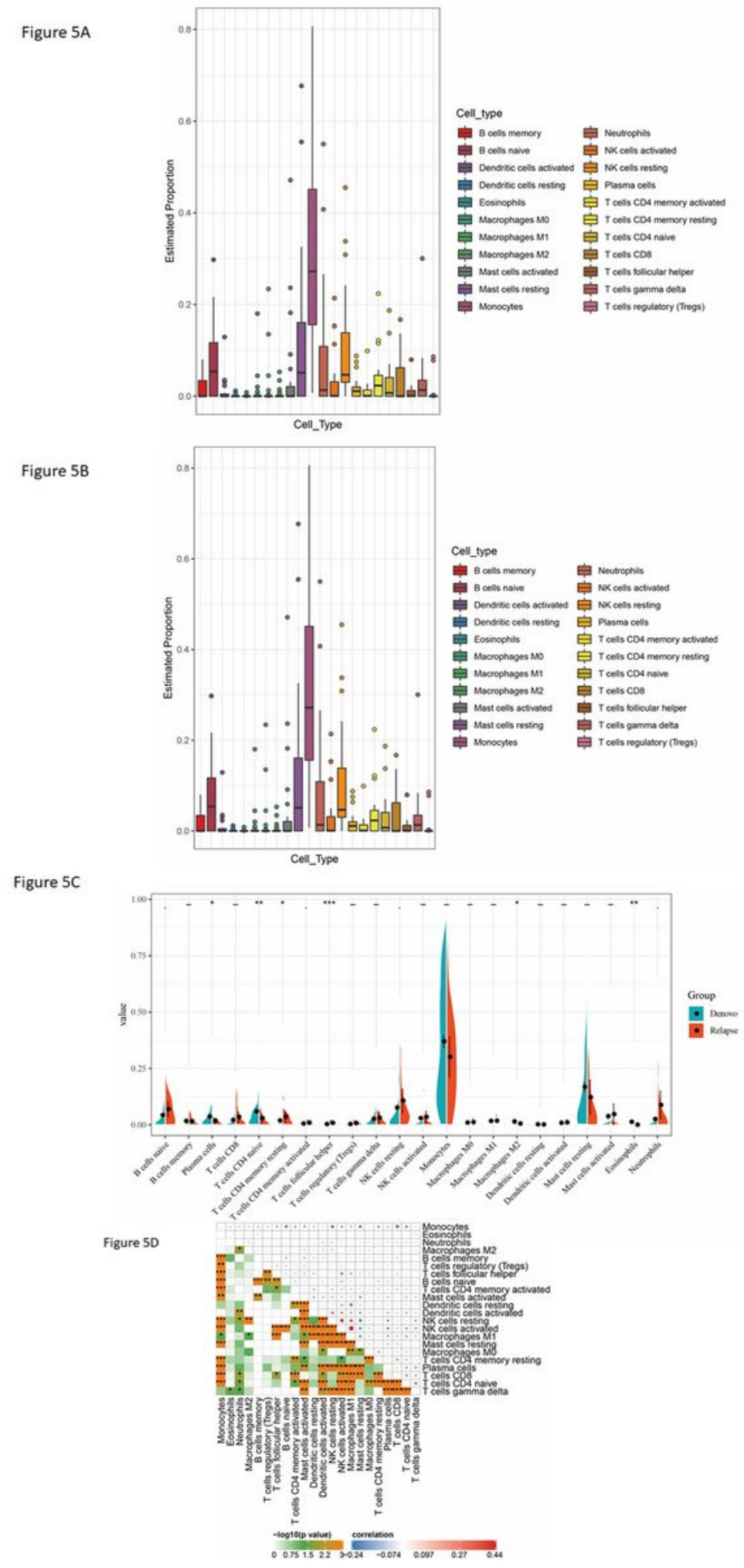

Figure 5

The landscape of immune infiltration in AML and difference of immune infiltration between denovo AML and relapse AML. A. denovo AML; B. relapse AML. C. Violin plot visualizing the differentially infiltrated immune cells; D. Correlation heatmap depicting correlations between infiltrated immune cells in AML 
Figure $6 \mathrm{~A}$

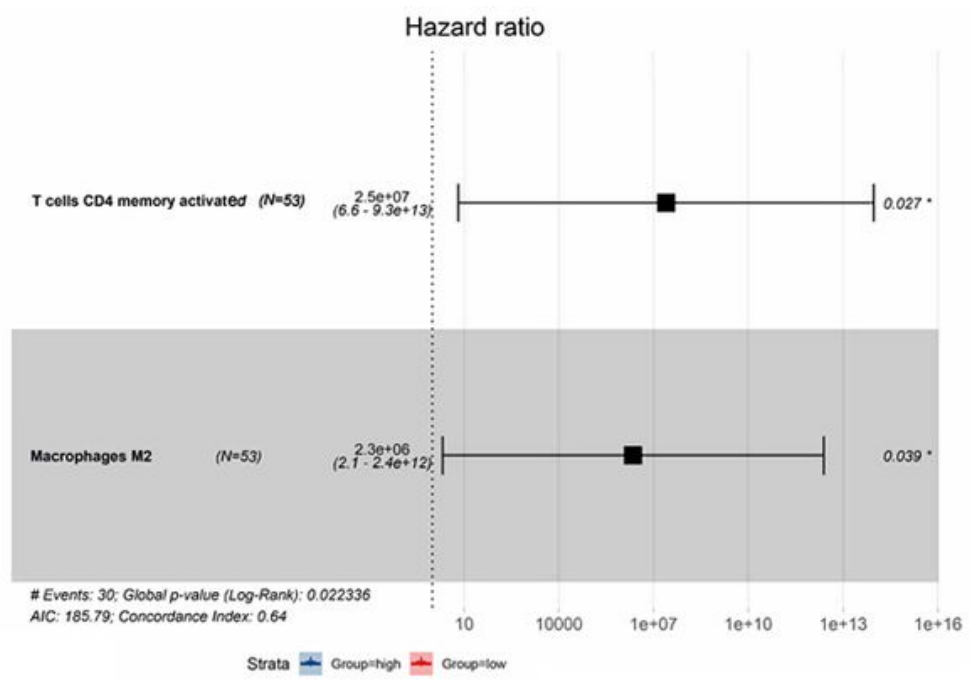

Figure 6B
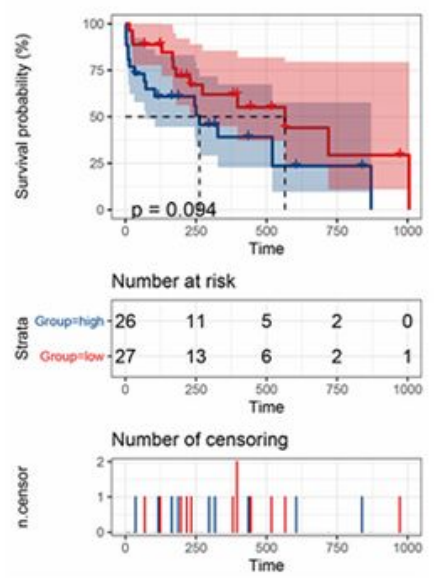

Figure $6 \mathrm{C}$

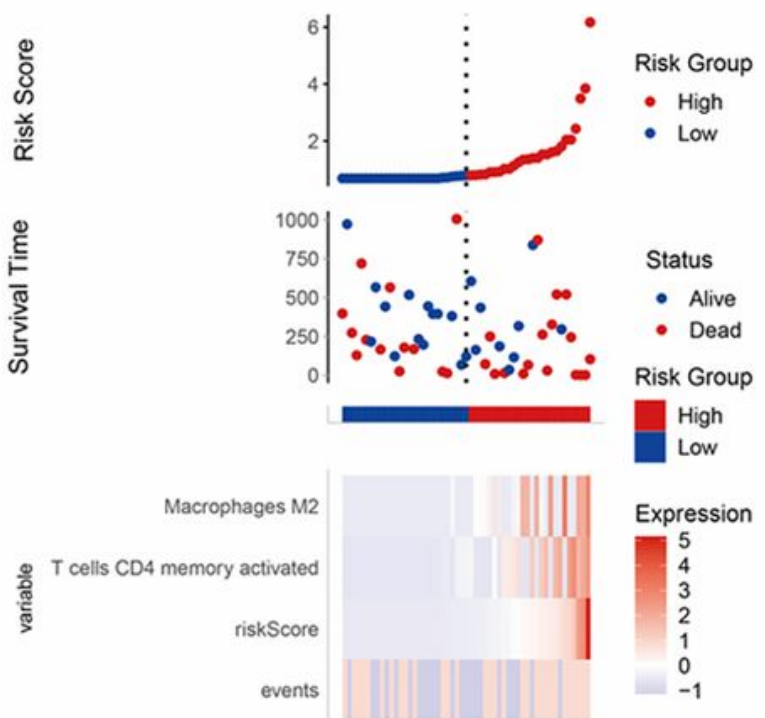

\section{Figure 6}

Clinical implications of TIICs subsets. A. Forest map of multivariate cox regression. B. Survival plots of median of immune cell subpopulations showed no statistical significance. C. The risk scores according to TIICs subsets for all patients in OHSU cohort are plotted in ascending order and marked as low risk (blue) or high risk (red), as divided by the threshold (vertical black line). 


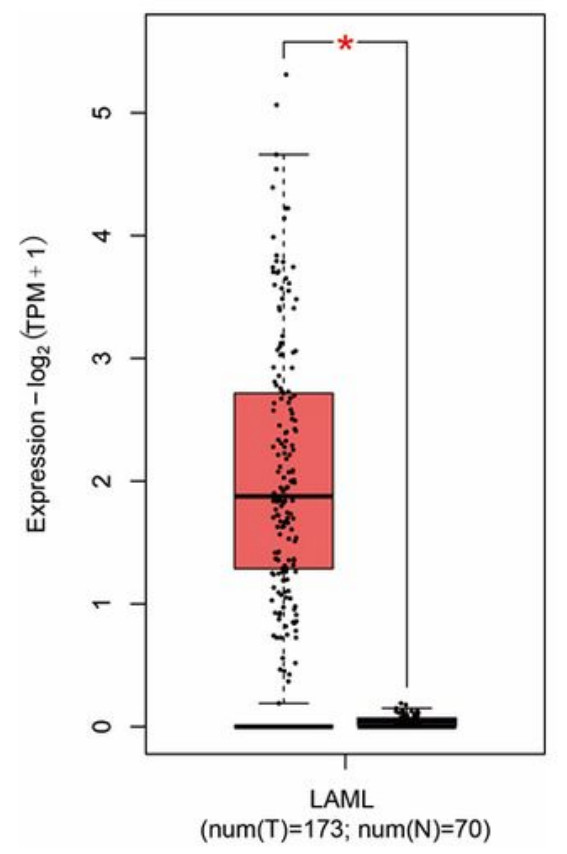

Figure 7A

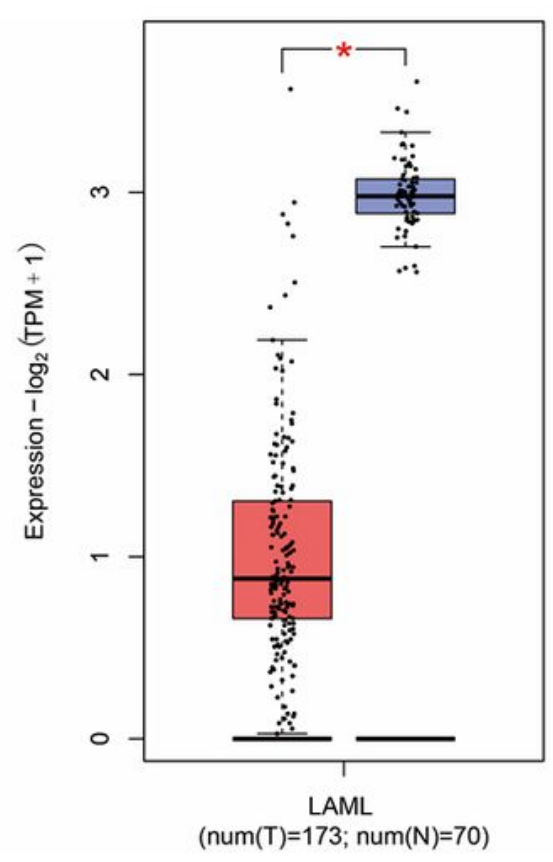

Figure 7B

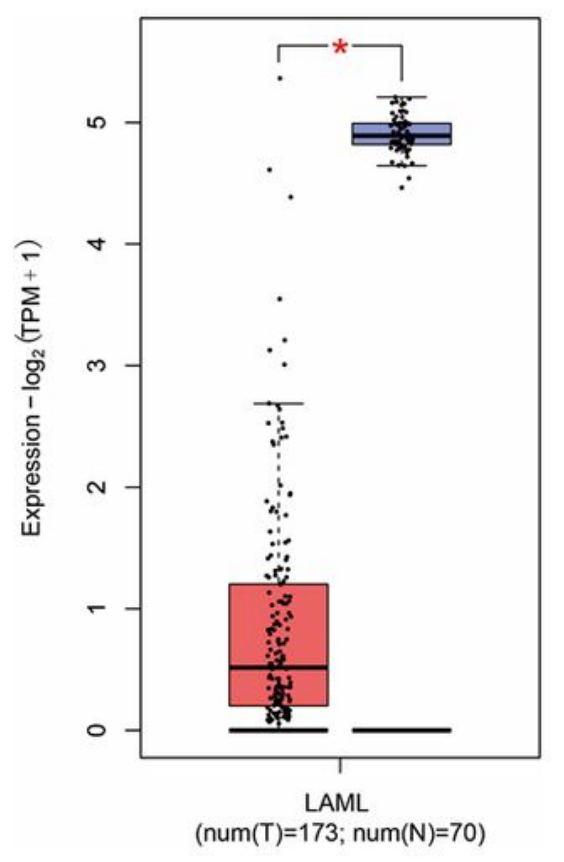

Figure $7 C$

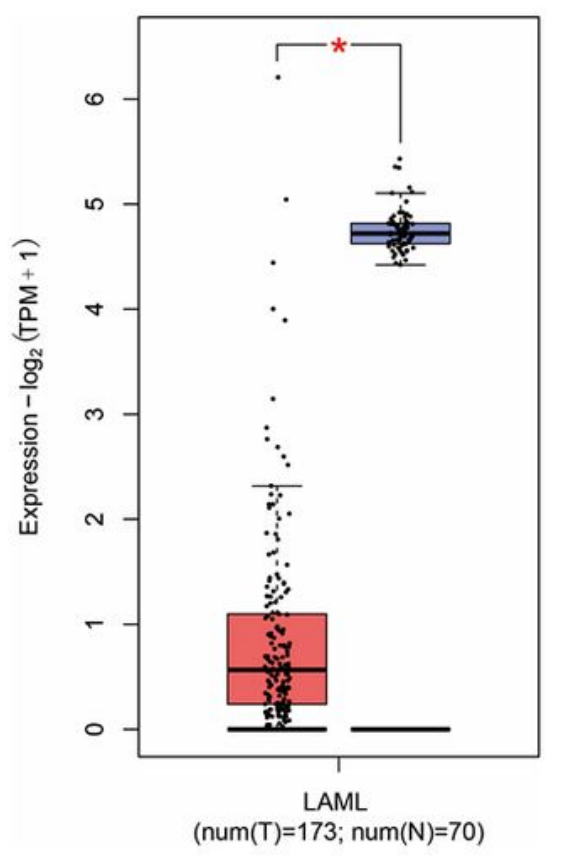

Figure 7D

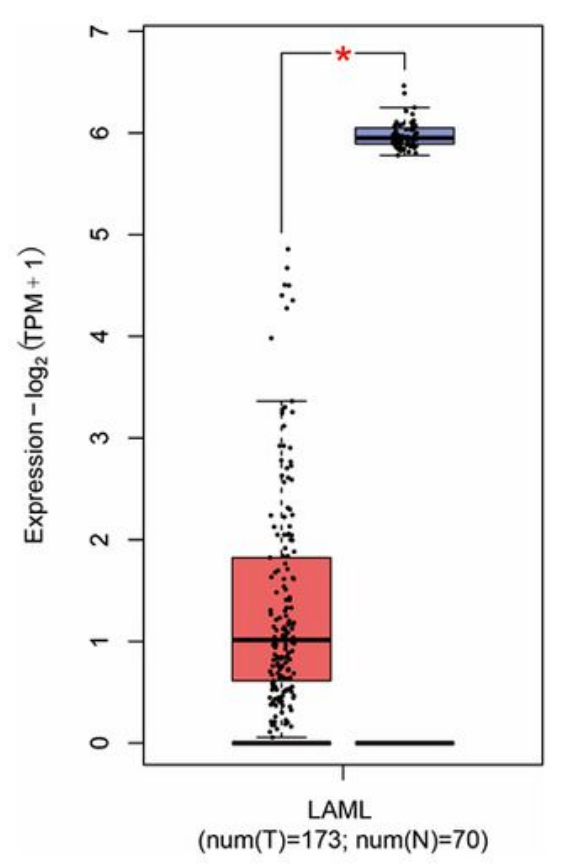

Figure 7E

\section{Figure 7}

The expression of the prognosis-related genes between AML patients and their normal samples. $A$. LRRN2 B. ALDH1L2 C. KLK1 D. PROS1 E. PSAT1. Red represents AML samples; Blue represents healthy control samples. 

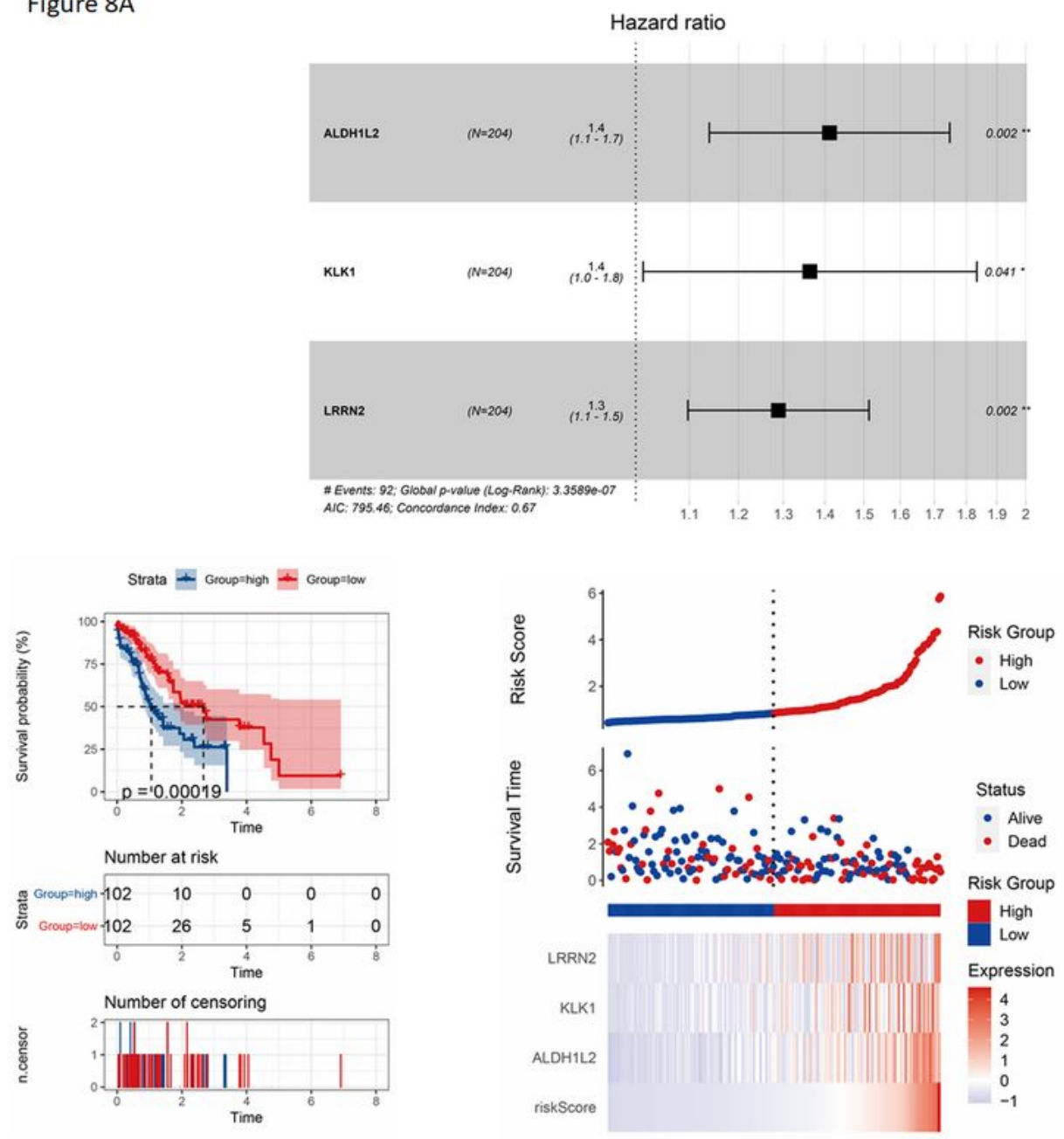

Figure 8B

Figure $8 C$

Figure 8D

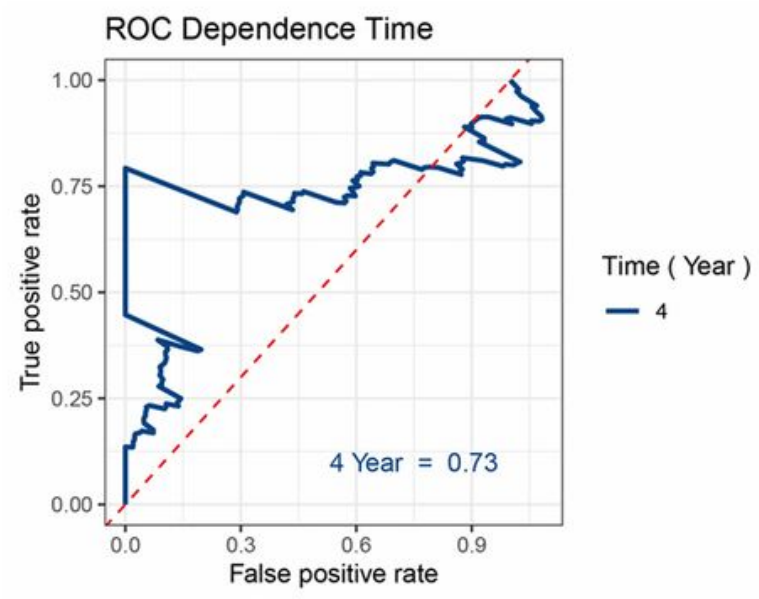

\section{Figure 8}

Survival prognosis model on the 3 prognosis-related genes. A. Forest map of multivariate cox regression. B. Survival analysis showed that the patients in the high-risk group had worse overall survival than those in low-risk group in OHSU cohort. C. The risk scores according to prognosis-related genes for all patients in OHSU cohort are plotted in ascending order and marked as low risk (blue) or high risk (red), as divided 
by the threshold (vertical black line). D. ROC analysis was performed to calculate the most optimal cutoff value to divide the $A M L$ patients into high risk and low risk group. 